(C) Elsevier, Paris

\title{
Editorial
}

\section{Génétique et psychiatrie : une nouvelle approche de l'étiopathogénie des maladies mentales ?}

\author{
M. LEBOYER et F. CLERGET-DARPOUX \\ Unité de génétique épidémiologique, INSERM U 155, Château de Longchamp, \\ Bois de Boulogne, 75016 Paris, France
}

(Reçu le 21-12-1987; accepté le 1-3-1988)

\begin{abstract}
Résumé - Jusqu'à présent, différentes méthodes ont été utilisées pour mettre en évidence l'influence de facteurs de risque d'origine génétique: comparaison du taux de concordance chez des jumeaux monozygotes et dizygotes, études d'adoption, analyse de ségrégation et analyse de linkage. Le développement explosif de la biologie moléculaire, fournissant un nombre grandissant de marqueurs génétiques, rend particulièrement prometteuse l'application des techniques de linkage. Néanmoins, il faut être conscient que l'application de cette stratégie aux maladies psychiatriques se heurte à des difficultés spécifiques: critères diagnostiques, âge de début variable, hétérogénéité probable, corrélations intrafamiliales environnementales.
\end{abstract}

génétique / étiopathogénie des maladies mentales

Summary - Genetics and psychiatry: towards a new etiopathology of psychiatric diseases? Until recently, different methods have been used to show the role of risk factors in the etiopathology of psychiatric diseases: comparison of concordance rates of monozygote and dizygote twins, adoption studies, segregation analysis, linkage analysis. This last approach has become an exciting one with the growing number of genetic markers due to progress in molecular genetics. Nevertheless, we must be aware of the fact that linkage strategy applied to psychiatric disease has to face specific problems: diagnostic criteria, variable age of onset, possible heterogeneity, environmental intrafamilial correlations.

genetics / etiopathology of psychiatric diseases

Les psychiatres français commencent à s'intéresser à la génétique épidémiologique et les généticiens à la psychiatrie. Pourquoi? Depuis 30 ans, la psychiatrie a bénéficié d'un renouvellement important de ses méthodes et de ses hypothèses. Au cours des années 70, le développement de la pharmacologie et de la biologie a permis de formuler des hypothèses sur le rôle que jouent les systèmes neurobiologiques dans les maladies psychiatriques. Cette approche s'est, jusqu'à présent, trouvée limitée dans ses moyens de vérifications. Mais le développement de la biologie moléculaire permet une approche nouvelle en offrant un outil supplémentaire à la génétique épidémiologique.
L'objectif de la génétique épidémiologique est la mise en évidence, pour une pathologie donnée, des différents facteurs de risque et plus particulièrement, ceux d'origine génétique. Elle s'intéresse à en préciser la nature, voire les mécanismes et éventuellement à déterminer le risque (c'est-à-dire la probabilité) pour un individu donné de développer une maladie. Cette discipline s'est d'abord intéressée à des pathologies à déterminisme simple et a mis au point, pour leur étude des méthodes d'analyse appropriées. En fait, la plupart des maladies actuellement étudiées, et en particulier les maladies psychiatriques, ne rentrent pas dans des schémas explicatifs aussi simples. Cela implique une modélisa- 
tion plus complexe tenant compte d'interactions possibles entre gènes, environnement et facteurs culturels. Il faut donc disposer de méthodes d'analyse nouvelles et d'informations supplémentaires. Celles-ci peuvent être apportées par l'étude des polymorphismes de l'ADN. Néanmoins, les problèmes spécifiques à la psychiatrie, critères diagnostiques, âge de début variable, hétérogénéité étiologique probable, poids des interactions familiales rendent plus difficiles encore l'utilisation et l'interprétation des méthodes d'analyse que nous allons tenter de présenter succinctement.

De nombreuses enquêtes épidémiologiques ont mis en évidence la concentration familiale de certaines maladies psychiatriques (agrégation familiale). L'étude classique permettant de montrer une telle concentration est la comparaison de la fréquence de la pathologie chez des apparentés (en général parents ou frères et sœurs) d'un groupe d'individus atteints d'une part, à des témoins appariés d'autre part.

L'existence d'une agrégation familiale suggère mais ne démontre pas le rôle de facteurs génétiques. En effet, elle peut être uniquement le résultat de la vie dans un environnement commun et/ou d'une interaction entre les membres d'une même famille. Après cette première étape, différentes approches sont utilisées pour la mise en évidence d'une composante génétique.

\section{Comparaison du taux de concordance chez des jumeaux monozygotes et dizygotes}

Un taux de concordance plus élevé chez les monozygotes que chez les dizygotes est un argument en faveur du rôle de facteurs génétiques, puisque les monozygotes, contrairement aux dizygotes, ont un patrimoine génétique identique. Insistons cependant sur le fait qu'une telle conclusion est basée sur l'hypothèse que la corrélation environnementale et culturelle chez les jumeaux est la même qu'ils soient monozygotes ou dizygotes.

La concordance est nettement plus élevée chez les monozygotes que les dizygotes dans la psychose maniaco-dépressive et dans la schizophrénie. Par ailleurs, le taux de concordance est légèrement plus élevé chez les dizygotes que chez les autres frères et sœurs. Ceci suggère que des facteurs génétiques d'une part et environnementaux d'autre part jouent un rôle dans le déterminisme de ces maladies.

\section{Etudes d'adoption}

L'idée qui soustend ce type d'études est que les composantes de milieu et les composantes génétiques sont dissociées par le processus d'adoption. Deux types de stratégies sont utilisées:

Dans la première, on constitue un échantillon de malades vivant dans une famille adoptive et on l'apparie avec un échantillon témoin d'adoptés non atteints. On compare alors les fréquences respectives de la maladie chez les parents biologiques des deux groupes. Dans l'autre stratégie, on prend deux échantillons de parents ayant donné leur enfant à adopter, le premier constitué de parents atteints, le second de parents sains. On compare alors la fréquence respective de la maladie chez les enfants adoptés pour chacun des 2 groupes. Si en plus les enfants sont classés en fonction du statut des parents adoptifs, on parle d'une étude de "cross-fostering". On peut alors comparer la fréquence respective de la maladie chez les enfants adoptés classés suivant le statut des parents biologiques et des parents adoptifs.

\section{Analyses de ségrégation}

Initialement, l'analyse de ségrégation avait pour but de tester si la ségrégation de la maladie (répartition de la maladie dans la famille) suivait les modes simples de transmission mendélienne: autosomale, lié à l'X, dominante ou récessive. Les maladies psychiatriques ne suivent pas des schémas de transmission aussi simples. De plus, la pénétrance est incomplète: un individu porteur du gène maladie n'exprimera pas toujours le phénotype maladie. Néanmoins, les méthodes d'analyse de ségrégation dont on dispose à l'heure actuelle permettent de tester des modèles génétiques plus complexes. Ces méthodes basées sur le principe du maximum de vraisemblance permettent de comparer des modèles entre eux. Les paramètres génétiques estimés sont ceux qui maximisent la vraisemblance des observations faites. Dans le cas de maladies psychiatriques, aucune analyse (voir pour revue Goldin et al., 1987) n'a permis la mise en évidence d'un gène majeur. Toutefois Goldin et al. (1983) ont montré que la taille moyenne nécessaire pour conclure à l'effet d'un tel gène pouvait être très élevée lorsque la pénétrance était faible et/ou lorsqu'il y avait hétérogénéité. 


\section{Difficultés d'applications et d'interprétations de ces méthodes en psychiatrie}

Un des problèmes essentiels à la recherche psychiatrique est l'établissement de critères diagnostiques permettant la classification des individus. Il est important d'utiliser des critères identiques d'une étude à l'autre et de savoir pour l'analyse où sera situé le seuil permettant de classer les individus en atteints ou non atteints. Par ailleurs, la plupart des maladies psychiatriques peuvent débuter à des âges différents et il faut tenir compte de ce facteur dans les analyses. De fait, la probabilité de développer ultérieurement une pathologie n'est pas la même pour un jeune enfant ou un adulte indemne au moment de l'observation. En outre dans toutes ces méthodes on pose un certain nombre d'hypothèses quant aux corrélations environnementales et culturelles entre individus apparentés. L'interprétation des résultats est, bien sûre, fonction de la validité de ces hypothèses. Par exemple, dans l'analyse de pistes faite dans la schizophrénie (Mac Gue et al., 1985), les auteurs ont conclu à une forte héritabilité génétique en imposant des contraintes sur les corrélations environnementales. Elles sont en particulier supposées identiques chez les jumeaux monozygotes et dizygotes. Quelle aurait été la conclusion si on n'avait pas imposé ces contraintes?

La grande diversité des formes cliniques que peut présenter une même maladie psychiatrique, la diversité des situations favorisant le déclenchement d'une pathologie et des âges de début laissent penser à une hétérogénéité étiologique soit des facteurs génétiques, soit des facteurs environnementaux, soit de leur association. Ceci peut expliquer que les méthodes d'analyse dont nous disposons actuellement, et qui sont conçues pour des modélisations simples, ne puissent rendre compte de la complexité des données observées en psychiatrie. L'existence potentielle d'une hétérogénéité étiologique et/ou de facteurs génétiques multiples constitue une difficulté méthodologique notable. La technique de linkage qui utilise l'information apportée par les marqueurs génétiques (polymorphismes de l'ADN) est d'un intérêt particulier, car elle permet de s'affranchir de certaines de ces difficultés.

\section{L'analyse de linkage}

Le but d'une analyse de linkage est de déterminer si un trait pathologique se transmet indépendam- ment ou non d'un marqueur génétique donné dans une famille comportant des individus atteints. Une transmission non-indépendante de la maladie et du marqueur génétique implique obligatoirement les 2 points suivants: 1) la participation d'au moins un gène dans l'étiopathogénie de la maladie; 2) ce gène est situé sur le même chromosome que le marqueur génétique. De fait, deux gènes situés sur des paires de chromosomes différents vont ségréger indépendamment. Au contraire, s'ils sont situés sur le même chromosome, ils auront tendance à se transmettre ensemble, ceci d'autant plus qu'ils sont proches l'un de l'autre. Plus ces locus sont proches, moins la probabilité de survenue de "crossingover" est grande et plus les enfants hériteront souvent de gamètes dits parentaux, en opposition aux gamètes dits recombinés. La distance entre les deux locus s'exprime en pourcentage de recombinaison (pourcentage de gamètes recombinés parmi un ensemble de gamètes transmis). Pour tester l'indépendance de transmission et estimer la distance entre deux locus, la méthode classique est la méthode des lod scores développée par Morton et al. en 1955. Cette méthode suppose connus les paramètres génétiques (fréquence génique et pénétrance) aussi bien au locus dit pathologique qu'au locus marqueur. Ceci n'est pas le cas pour les maladies psychiatriques. On a cependant montré qu'une erreur de modélisation au locus pathologique pouvait biaiser fortement l'estimation du taux de recombinaison, mais ne pouvait simuler un linkage qui n'existait pas (Clerget-Darpoux et al., 1986).

Les marqueurs génétiques sont donc potentiellement les meilleurs outils pour la mise en évidence de composantes génétiques dans les maladies psychiatriques. Cependant, pour apporter une information, un marqueur génétique doit être polymorphe. En effet, pour montrer que le gène pathologique et le gène marqueur sont transmis ensemble, il faut pouvoir distinguer chacun des deux chromosomes de la paire: celui qui porte l'allèle pathologique et celui qui porte l'allèle sain. Il faut donc que le marqueur s'exprime différemment sur chacun de ces chromosomes. En d'autres termes, que l'un des deux parents soit un double héterozygote au locus marqueur et au locus maladie. Il est par conséquent nécessaire d'utiliser des gènes marqueurs qui montrent un grand polymorphisme dans la séquence d'ADN. Il y a quelques années encore, les marqueurs génétiques existant étaient en nombre très réduits et peu polymorphes. Actuellement, grâce aux techniques de biologie molécu- 
laire, le nombre de polymorphismes identifiés augmente de manière explosive. Deux stratégies peuvent être employées: 1)l'utilisation de marqueurs choisis au hasard; 2) des marqueurs génétiques dits "fonctionnels», c'est-à-dire, codant pour des éléments participant aux systèmes de neurotransmission et donc potentiellement impliqués dans l'étiopathogénie des maladies psychiatriques.

A titre d'illustration, une équipe américaine (Egeland et al., 1987) a mis en évidence dans un grand pedigree Amish, une ségrégation nonindépendante du marqueur Harvey-ras, situé sur le bras court du chromosome 11 , et de la maladie maniaco-dépressive. Or, le marqueur Harvey-ras est très proche du gène de la tyrosine hydroxylase codant pour l'enzyme de l'étape limitante de la synthèse des catécholamines. A la lumière de l'hypothèse catecholaminergique de la dépression, ces résultats préliminaires paraissent très prometteurs (Mallet et Leboyer, 1987). Néanmoins, ils doivent être interprétés avec prudence. Tout d'abord le seuil de signification statistique donné dans l'étude peut être discuté: 1) Il n'est pas tenu compte de l'analyse simultanée de plusieurs marqueurs génétiques (problème de signification des tests multiples). 2) La définition du seuil entre atteint et non atteint n'est pas claire. Les psychoses maniaco-dépressives, les dépressions unipolaires, les psychoses schizoaffectives, les psychoses atypiques appartiennent-elles à la même catégoric nosographique? 3) Le modèle utilisé dans l'analyse (modèle autosomal dominant à pénétrance incomplète) est très discutable. 4) Enfin, à ce jour, aucune étude n'a pu répliquer ce résultat. Ceci est expliqué par la plupart des auteurs, comme témoignant d'une hétérogénéité génétique: il existerait un ou plusicurs gènes déterminant la susceptibilité génétique à la maladic.

Cette hétérogénéitć est argumentéc par les études de Risch et Baron (1984) et de Mendlewicz et al. (1987) montrant un linkage avec les marqueurs du chromosome $X$ dans certaines familles de psychoses maniaco-dépressives et pas dans d'autres. Malgré ces réserves, il est fondamental que de telles études soient répliquées ce qui serait la meilleure façon de lever l'ambiguïté sur la signification des résultats déjà obtenus.

Il est bien clair que l'hypothèse sous-jacente aux études envisagées ici n'est pas celle d'un contrôle des maladies psychiatriques par l'effet simple et unique d'un gène. Bien au contraire, les études actuelles convergent toutes vers l'idée d'une étiologie complexe où interviennent à la fois des facteurs génétiques et environnementaux. La mise en évidence de facteurs génétiques pourrait, cependant, permettre de micux mesurer pour chaque individu son risque vis-à-vis de facteurs environnementaux susceptibles soit de déclencher, soit de protéger de la survenue d'une maladie donnée.

\section{Références}

Clerget-Darpoux F., Bonaiti C. \& Hochez J. (1986) Effects of misspecifying genetic parameters in lod score analysis. Biometrics 42, 393-399

Egeland J., Gerhard D.S., Pauls D., Sussex J.N., Kidd K.K., Allen C.R., Hostetter A.M. \& Houseman D.E. (1987) Bipolar affective disorders linked to DNA markers on chromosome 11. Nature 325, $783-787$

Goldin L.R., Gershon E.S., Targum S.D. \& McGinnis M. (1983) Segregation and linkage analyses in families of patients with bipolar, unipolar, and schizoaffective mood disorders. Am. J. Hum. Genet. 35, 274-287

Goldiu 1..R. \& Gershon E.S. The genetic epidemiology of major depressive illness. Am. Psychiatric Assoc. Ammu. Rev. vol. 7 (in press)

McGue M., Gottesman I. \& Rao D). (1985) Resolving genetic models for the transmission of schizophrenia. Genet. Epidemiol. 2, 99-110

Mallet J. \& Leboyer M. (1987) Molecular genetics, bipolar disorders and the catecholamine hypothesis. Symposium, Biological Psychiatry Today

Mendlewicz J., Simon P., Sevy S., Charon F., Brocas H., Legros S. \& Vassart G. (1987) Polymorphic DNA marker on $X$ chromosome and manic depression. Lancel 30, 1230-1231

Risch N. \& Baron M. (1982) X-linkage and genetic heterogeneity in bipolar-related major affective illness : reanalysis of linkage data. Ann. Hum. Genet. $46,153-166$ 